\title{
EFFECTS OF FLUOXETINE ON WEIGHT GAIN AND FOOD INTAKE IN SMOKERS WHO REDUCE NICOTINE INTAKE
}

\author{
Ovide F. Pomerleau, Cynthia S. Pomerleau, \\ ERIC M. MORRELL, and JOHN M. LOWENBERGH \\ Behavioral Medicine Program, Department of Psychiatry, University of Michigan, \\ Ann Arbor, Michigan, U.S.A.
}

(Received 10 August 1990; in final form 13 December 1990)

\begin{abstract}
SUMMARY
The effect of fluoxetine hydrochloride, a 5-HT uptake inhibitor (60 mg/day PO), in preventing weight gain associated with nicotine reduction was investigated in participants in a double-blind, placebo-controlled smoking-cessation trial. A lunch of cheese pizza and chocolate bars was offered, and caloric intake was monitored. The analysis focused on subjects (placebo: $n=11$; fluoxetine: $\mathrm{n}=10$ ) who succeeded in reaching cotinine levels of less than $50 \%$ of their starting cotinine levels (signifying a stringent reduction in nicotine intake) and who participated in pre- and post-nicotine reduction lunch sessions 70 days apart. Subjects on placebo gained significantly more weight (mean $\pm S E M=+3.3 \pm 0.7 \mathrm{~kg}$ ) than subjects on fluoxetine $(-0.6 \pm 1.2 \mathrm{~kg}$ ). In fluoxetine-treated subjects, weight gain/loss was strongly correlated with initial body mass index, with higher BMI being associated with greater decreases in weight. A trend towards decreased caloric intake in the fluoxetine group was observed; the change in total calories at lunch was significantly correlated with weight change, an association accounted for principally by change in pizza intake. We conclude that fluoxetine treatment effectively prevents the weight gain that accompanies nicotine reduction and that this phenomenon is mediated, at least in part, by diminished caloric intake.
\end{abstract}

\section{INTRODUCTION}

On average, cigarette smokers weigh less than comparably aged non-smokers, and many smokers who quit smoking gain 3-5 kg in the year following cessation (Pomerleau et al., 1978; Grunberg, 1986a; Klesges et al., 1989). Animal studies have established an inverse doseresponse relationship between nicotine, chronically administered over a period of months, and body weight (Bowen et al., 1986; Grunberg, 1986b). Though conflicting results have been reported (e.g., Hughes et al., in press), research on nicotine replacement tends to corroborate these findings: Subjects who quit smoking have been reported to gain less weight when they consistently used nicotine polacrilex (Fagerstrom, 1987; Killen et al., 1990), a phenomenon that is inversely related to dosage (Stitzer \& Gross, 1988). Weight gain is seen not only in those who abstain completely, but also in smokers who substantially reduce cigarette consumption (Perkins et al., 1987).

Address correspondence and reprint requests to: Dr. Ovide F. Pomerleau, Behavioral Medicine Program, University of Michigan, Department of Psychiatry, Riverview Building, 900 Wall Street, Ann Arbor MI 48105, USA. 
The extent to which post-cessation weight gain is related to changes in appetite, specific taste preferences, and/or actual food intake, as opposed to changes in basal metabolism or activity level, remains unclear. Some studies have suggested that nicotine administration reduces food intake (McNair \& Bryson, 1983), with a selective effect on sweet-tasting foods (Grunberg, 1982; Hall et al., 1989) as well as on taste hedonics (Perkins et al., 1990b); nicotine withdrawal has been shown to enhance caloric intake (Hatsukami et al., 1984; Stamford et al., 1986) and intake of sweets (Perkins et al., 1990a). Other studies, however, have failed to reveal any effects of nicotine on food intake in rats (Wellman et al., 1986); human studies also have identified no differences in food intake between smokers and nonsmokers (Albanes et al., 1987) and no increases in caloric intake following withdrawal (Rodin, 1987). Likewise, Gilbert and Pope (1982) did not observe any shift in preference for sweet foods following withdrawal. There is even evidence in the animal literature to suggest that chronic nicotine exposure enhances appetite for sucrose (Jias \& Ellison, 1990).

Fluoxetine (Prozac ${ }^{\circledR}$ ), a selective serotonin uptake inhibitor, also has been reported to decrease eating and to produce weight loss reliably in controlled clinical trials (Levine et al., 1987); these effects have been linked to a reduction in "carbohydrate craving" (Ferguson \& Feighner, 1987), though not specifically to sweet-tasting carbohydrates. Accordingly, the present investigation took advantage of a placebo-controlled clinical trial of fluoxetine as an adjunct to behavioral smoking cessation treatment to examine the question of how fluoxetine might affect weight and intake of foods in the context of decreased nicotine intake. The study involved offering the participants a lunch, in a controlled laboratory setting, of two commonly eaten high-carbohydrate foods, one sweet-tasting (chocolate) and one not (cheese pizza), on five different occasions. We hypothesized that (1) fluoxetine would prevent the weight gain commonly seen in smokers who cut down or eliminate nicotine intake, and that (2) subjects on fluoxetine would decrease food intake compared to subjects on placebo. We also were interested in determining whether the intake of a sweet-tasting food, chocolate, would be differentially affected.

\section{SUBJECTS AND METHODS}

\section{Subjects}

Subjects were smokers recruited from the local community to participate in a randomized, placebo-controlled, double-blind clinical trial of fluoxetine as an aid to smoking cessation. Subjects were required by the sponsor's protocol to be at least 18 years of age, to have a history of smoking 20 or more cigarettes per day for at least a year, to have an educational level sufficient to permit intelligent communication with study personnel, and to be willing to quit smoking by a stated time in the study. Exclusion criteria were previous participation in any fluoxetine study; pregnant or lactating women, and women of childbearing age not using a medically accepted means of contraception; regular use of psychotropic drugs or smoking cessation medications; regular or intermittent use of smokeless tobacco; history of bipolar depression; or any condition judged to be medically unstable.

Fifty smokers, 30 female and 20 male, were originally enrolled in the trial. Thirty-seven remained in the study at the time the current data were collected. Two fluoxetine subjects had withdrawn because of drug side effects (allergic reaction/rash; severe fatigue), and a third fluoxetine subject was eliminated because of a protocol violation (he tried to halve his dosage because of an anxiety reaction attributed to the drug). One placebo subject had been eliminated because she had become pregnant. The nine remaining dropouts all cited schedule conflicts or stress as their reasons for withdrawal. Compliance with drug-administration procedure was monitored by requiring patients to turn in their empty bottles before new supplies were issued and was confirmed subsequently by assays of serum fluoxetine.

Since the focus of interest in the present study was upon the effects of fluoxetine in the context of nicotine 
withdrawal rather than upon treatment outcome, we restricted our analysis to subjects who succeeded in reaching cotinine levels of less than half their baseline levels (indicating a stringent reduction in nicotine intake) by the test session at which they were expected to achieve abstinence (Session 4). Data for two subjects were deleted from the analysis, because both their baseline and test Session 1 (pre-randomization, pre-reduction) cotinine values were below $50 \mathrm{ng} / \mathrm{ml}$. (The nicotine dependence criterion for admission to the study was based on number of cigarettes per day rather than on Fagerstrom Tolerance Questionnaire scores or cotinine values.) Of the 23 subjects (11 on placebo and 12 on fluoxetine) who met the criterion for cotinine reduction, two were unavailable to participate in the fourth lunch session for reasons unrelated to the study. Thus, complete data for the first and fourth sessions were available only for 21 subjects -11 on placebo and 10 on fluoxetine. (Seven subjects in each group achieved final cotinine values of $0 \mathrm{ng} / \mathrm{ml}$; cotinine values for the remaining subjects ranged from 29 to $138 \mathrm{ng} / \mathrm{ml}$.) Demographic and baseline data for all subjects included in this analysis are shown in the Table.

\section{SUBJECT CHARACTERISTICS (MEAN \pm SEM)}

\begin{tabular}{lcc}
\hline & Placebo Subjects & Fluoxetine Subjects \\
\hline $\mathrm{N}$ & 11 & 10 \\
\% Female & $64 \%$ & $70 \%$ \\
Age (years) & $47.4 \pm 10.7$ & $43.9 \pm 10.8$ \\
Baseline Cotinine (ng/ml) & $255.9 \pm 125.8$ & $250.7 \pm 105.0$ \\
Final Cotinine (ng/ml) & $19.7 \pm 28.2$ & $22.8 \pm 45.2$ \\
Fagerstrom TQ (range: 0-11) & $6.2 \pm 1.2$ & $6.0 \pm 1.4$ \\
Cigarettes/Day & $27.5 \pm 4.8$ & $30.0 \pm 1.4$ \\
Baseline Weight (kg) & $75.2 \pm 16.6$ & $67.3 \pm 11.7$ \\
Height (m) & $1.66 \pm 0.1$ & $1.64 \pm 0.1$ \\
Baseline Body Mass Index (BMI) & $27.2 \pm 5.3$ & $25.1 \pm 3.5$ \\
(weight $\div$ height ${ }^{2}$ ) & & \\
\hline
\end{tabular}

\section{Procedure}

Subjects were exposed, in groups of approximately 10 individuals, to a minimal behavioral intervention for smoking. The treatment manual, "Freedom from Smoking" (American Lung Association, 1980), was used. Only cursory advice on exercise and dieting was offered. Weight was measured at each treatment visit. Groups met from $1000 \mathrm{~h}$ to $1130 \mathrm{~h}$; eating test Sessions $1-5$ immediately followed selected treatment visits and were carried out, respectively, on Days 7 (the day before randomization to fluoxetine, $60 \mathrm{mg} /$ day PO, or placebo), 21, 35,77 , and 161. (These numbers represent target dates; for some subjects, actual session dates varied from a day to a week.) On the day of the second test session, subjects were instructed to cut down smoking, and on the day of the third test session, they were instructed to quit smoking altogether. Thus, by the time of the fourth eating test session (10 weeks post-randomization), subjects were expected to have reached a point of stable abstinence while maintaining drug administration; the same conditions were in effect at the fifth eating test session.

During each eating test, subjects were offered a standard lunch consisting of a small cheese pizza (approximately $800 \mathrm{~g}$ ), a large Cadbury's chocolate bar (either $149 \mathrm{~g}$ or $207 \mathrm{~g}$ ) broken into bite-sized squares, and cold water. Nutrient content for $1 \mathrm{~g}$ of pizza was estimated to be $0.33 \mathrm{~g}$ carbohydrate, $0.07 \mathrm{~g}$ fat, $0.12 \mathrm{~g}$ protein, and $0.46 \mathrm{~g}$ water, with a total of $2.43 \mathrm{kcal}$; for $1 \mathrm{~g}$ chocolate (according to data provided by the manufacturer), $0.61 \mathrm{~g}$ carbohydrate, 0.29 fat, $0.08 \mathrm{~g}$ protein, and 0.01 water with a total of $5.39 \mathrm{kcal}$ (Pennington, 1989). Subjects ate in groups of approximately 10 , which included a random mix of fluoxetine and placebo subjects, and were instructed not to talk to one another during the lunch. The pizza and chocolate bars for each subject 
were weighed before and after lunch. Instructions for carrying out eating test session sequences were taped to ensure standardization, and "mood music" was played when instructions were not being presented. No restrictions were placed on the time allocated for eating. Before and after lunch, subjects were given visual analogue scales to assess anticipation of and satisfaction derived from eating. After lunch, subjects also were queried about possible nausea during lunch and whether they were restricting eating for purposes of weight reduction. Subjects were permitted to read magazines upon completing the meal but were not dismissed until several minutes after the last subject had completed the last questionnaire, in order to prevent time considerations from influencing eating behavior.

\section{Data analysis}

Because of further subject attrition by Session 5, because of missing lunch data in Sessions 2 and 3, and because the inevitable variability of nicotine/cotinine levels during the cutting-down stage would further complicate interpretation, a decision was made to analyze only the data from the first and fourth eating test sessions. Analyses included independent $t$-tests on change scores between the first and fourth test sessions and correlations between change in weight and change in food intake. In instances in which directional hypotheses were specified, one-tailed statistical tests were used.

\section{RESULTS}

The 21 subjects included in the analysis did not differ significantly, by two-tailed independent $t$-tests, from the 29 other subjects who originally enrolled in the trial with respect to any of the following baseline variables: age, weight, height, body mass index (BMI = weight $\div$ height ${ }^{2}$ ), total Fagerstrom TQ score, or gender composition. Likewise, no significant differences in food intake at the first eating session were detected.

The groups did not differ significantly, by two-tailed independent $t$-tests, on any of the baseline characteristics shown in the Table. Most subjects were of normal weight, though four (three on placebo and one on fluoxetine) had a BMI in excess of 30, categorizing them as overweight. No significant differences in nausea or restrictive eating were detected between the two groups for either test session. Changes in anticipation of eating and of postprandial satisfaction between the two test sessions did not differ significantly between the two groups. Although subjects were blinded to their drug status, 8 of the 11 placebo subjects and 7 of the 10 fluoxetine subjects were able to guess correctly at the time of the second test session. (Except for one fluoxetine subject who thought she was on placebo, the remainder said they did not know.) No feedback was given regarding the correctness of subjects' guesses about which study drug they were taking; indeed, none was available, since the study was run double-blind. No subject ate either all the pizza or all the chocolate portions at any test meal.

All but one of the placebo subjects gained weight; five fluoxetine subjects lost weight, one remained unchanged, and four gained weight. Mean weight change for the two groups is shown in the Figure. Weight change from the pre-nicotine reduction test session (Session 1) to the post-nicotine reduction test session (Session 4) differed significantly, with the placebo group posting a weight increase of

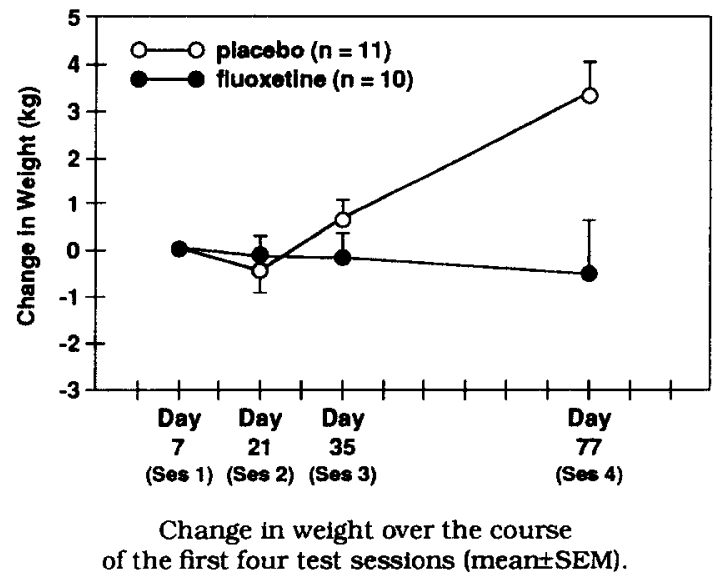


$3.3 \pm 0.7 \mathrm{~kg}$ and the fluoxetine group showing a decrease of $0.6 \pm 1.2 \mathrm{~kg}(t[19]=3.02, p=0.000$, one-tailed).

In an attempt to determine the extent to which observed changes in weight were related to initial weight status, change in weight was related to BMI separately for the two groups. In the fluoxetine group, baseline BMI was strongly negatively correlated with weight change ( $r=-.95$, $p=0.000$, two-tailed), higher BMI being associated with greater weight loss. Weight change in the placebo group was positively, but not significantly, correlated with baseline BMI $(r=+.23$, $p=$ NS, two-tailed).

With regard to changes in total calories consumed at lunch between the fourth session and the first session, subjects in the fluoxetine group showed a trend $(t[19]=1.39, p=0.089$, onetailed) towards reduced consumption $(-181 \mathrm{kcal})$ compared to subjects on placebo $(+10 \mathrm{kcal})$. The fluoxetine group consumed 172 fewer kcal of pizza and 9 fewer kcal of chocolate, whereas the placebo group consumed 48 fewer kcal of pizza but 58 more kcal of chocolate. Changes in intake of the individual foods did not differ significantly, nor did changes in the ratio of chocolate to pizza caloric intake.

In order to investigate further the question of whether the observed weight changes might be related to changes in food intake, correlations between weight change and change in intake were computed for the entire sample of 21 subjects, comparing Session 4 with Session 1. Change in total lunch calories were significantly correlated with weight change $(r=+.44$, $p=0.048$, two-tailed); this association was principally accounted for by changes in intake of pizza $(r=+.49, p=0.026$, two-tailed); no correlation was detected for change in chocolate intake $(r=+.09, p=\mathrm{NS}$, two-tailed).

\section{DISCUSSION}

There were no baseline differences between the entire sample and the subjects eliminated from the analyses. Likewise, no differences were detected between the two groups.

Subjects showed clear-cut differences in weight-change profiles over the 10 weeks that elapsed between eating test Sessions 1 and 4, with fluoxetine-treated subjects exhibiting a slight decline and placebo-treated subjects gaining more than $3 \mathrm{~kg}$. The weight gain for the placebo subjects is in keeping with the magnitude of changes reported after stringent nicotine reduction (Klesges et al., 1989).

Over the same interval, lunch food intake showed a trend toward diminished consumption, particularly in pizza. Changes in eating showed a significant positive correlation with changes in weight in individual subjects, suggesting that prevention of weight gain by fluoxetine may have been related, at least in part, to decreased food consumption. Our correlation of +.44 is strikingly similar to the correlation of +.41 reported by Spring et al. (1991) between weight change and caloric intake, examined under somewhat more naturalistic conditions in a combined sample of obese female subjects who stopped or drastically reduced smoking while being treated with placebo or another serotonergic compound, d-fenfluramine (a 5-HT releaser and uptake inhibitor). Neither our study nor that of Spring et al., however, rules out the possibility of additional fluoxetine effects on basal metabolism or physical activity. Blundell (1986), in a review of serotonin manipulations on the structure of eating behavior, pointed out that enhancement of serotonin neurotransmission typically results in a reduction in meal size and a curtailment of eating rate, consistent with the entrainment of satiety. The present findings, based on total caloric intake of a standardized lunch, are in keeping with the observation of decreased meal size; unfortunately, eating rate was not monitored, so the impact of fluoxetine 
on this variable cannot be ascertained. The lack of significant differences between the fluoxetine and placebo groups in either anticipation of eating or satisfaction from the meal fails to support the possibility that fluoxetine affects awareness of hunger or satiety; a larger sample and a different design would be required to settle this issue definitively.

The present study does not resolve the question of whether fluoxetine differentially affected intake of sweet-tasting food (Spring et al., 1987). Placebo-treated subjects consumed more chocolate in Session 4 than in Session 1, but the order in which the foods were consumed was neither controlled nor monitored, and the nutrient composition between the two foods varied in other respects as well (e.g., fat content). The main findings of reduced caloric intake and prevention of weight gain in subjects taking fluoxetine, however, are consistent with previous reports of decreased appetite and weight loss in overweight nonsmokers treated with fluoxetine (Ferguson \& Feighner, 1987; Levine et al., 1987). They also are supported by the findings of Spring et al. (1991), who observed that placebo-treated subjects increased carbohydrate intake and gained weight, whereas d-fenfluramine-treated subjects returned quickly to baseline carbohydrate intake levels and lost weight. Our results, however, will need to be replicated in larger samples, with presentation of a larger variety of food choices, systematic variation of nicotine and fluoxetine dosing, and more rigorous control over such experimental and subject variables as fasting status, caffeine intake, and baseline weight. Differences in other factors that might have affected eating behavior, such as nausea and restrictive eating, were not observed in our small sample but cannot be ruled out altogether. Of particular importance are understanding the effects of fluoxetine on sweet taste preference and metabolism in both normal-weight and obese individuals, as well as obtaining a better definition of actual eating behavior over a larger sampling interval.

Some investigators have held that fear of weight gain may serve as a motive for continued smoking (Shor et al., 1981; Sorensen \& Pechacek, 1987) and that concem about weight gain deters some smokers from attempting to quit (Klesges \& Klesges, 1988). Certainly, for those smokers who have shown large increases in weight in previous quit attempts or who are daunted by the prospect of gaining weight, adjunctive treatment to prevent weight gain may diminish some of the unpleasantness of smoking cessation. Different approaches are currently available for this purpose. For example, formal behavior modification procedures for weight control have been added to smoking treatment. There is some evidence, however, that the combination of two intensive behavioral treatments may not be efficacious and may even be counter-productive (Sharon Hall, personal communication). Nicotine polacrilex has been reported to help prevent weight gain after quitting cigarettes. In a study by Stitzer and Gross (1988), for example, the use of nicotine gum kept the weight gain of the treatment group $(+1.7$ $\mathrm{kg}$ ) to little over half that of the placebo group; subjects who made the greatest use of the gum (chewing a mean of nine pieces per day) gained the least $(+0.7 \mathrm{~kg})$. On the other hand, Hughes et al. (in press), in a large nicotine gum trial, failed to observe prevention of weight gain; the investigators speculated that self-dosing had been inadequate to maintain weight control. Such findings raise the question of whether a higher nicotine replacement dosage is needed for weight management than for stopping smoking; if so, the implications of using doses of nicotine in excess of the minimum required to promote smoking cessation for the sole purpose of controlling weight must be carefully reviewed.

Taking these considerations into account, we conclude that treatment with fluoxetine is as efficacious as any technique for preventing post-smoking cessation weight gain that has been tried to date and has the advantage that compliance with the procedure/dosing may be more readily achieved. The drug may prove especially helpful in countering weight gain pursuant to 
nicotine withdrawal in obese smokers, as suggested by the strong relationship between BMI and weight change in the fluoxetine-treated subjects. Our findings, if corroborated, may serve as a prototype for the specific use of pharmacological techniques to deal selectively with important components in the sequence of changes and adjustments that go with giving up smoking.

Acknowledgments: This investigation was conducted as an adjunct to a clinical trial, "Fluoxetine vs Placebo: Fixed Dose in Smoking Withdrawal," Protocol B1Y-MC-HCDI, sponsored by Eli Lilly and Company.

\section{REFERENCES}

Albanes D, Jones Y, Micozzi MS, Mattson ME (1987) Associations between smoking and body weight in the U.S. population. Amer J Publ Health 77: 439-444.

American Lung Association (1980) Freedom from Smoking. American Lung Association, New York.

Blundell JE (1986) Serotonin manipulations and the structure of feeding behaviour. Appetite 7: 39-56.

Bowen DJ, Eury SE, Grunberg NE (1986) Nicotine's effects on female rats' body weight: caloric intake and physical activity. Pharmacol Biochem Behav 25: 1131-1136.

Fagerstrom KO (1987) Reducing the weight gain after stopping smoking. Addict Behav 12: 91-93.

Ferguson JM, Feighner JP (1987) Fluoxetine-induced weight loss in overweight non-depressed humans. Int J Obes 11: $163-170$

Gilbert RM, Pope MA (1982) Early effects of quitting smoking. Psychopharmacology 78: 121-127.

Grunberg NE (1982) The effects of nicotine and cigarette smoking on food consumption and taste preferences. Addict Behav 7: 317-331.

Grunberg NE (1986a) Behavioral and biological factors in the relationship between tobacco use and body weight. In: Katkin ES, Manuck SB (Eds) Advances in Behavioral Medicine (Vol 3). JAI Press, Greenwich CT, pp 97-129.

Grunberg NE (1986b) Nicotine as a psychoactive drug: appetite regulation. Psychopharmacol Bull 22: 875881.

Hall SM, McGee R, Tunstall C, Duffy J, Benowitz N (1989) Changes in food intake and activity after quitting smoking. J Consult Clin Psychol 57: 81-86.

Hatsukami DK, Hughes JR, Pickens RW, Svikis D (1984) Tobacco withdrawal symptoms: an experimental analysis. Psychopharmacology 84: 231-236.

Hughes JR, Gust SW, Skoog K, Keenan, R, Fenwick JW (in press) Symptoms of tobacco withdrawal: a replication and extension. Arch Gen Psychiatry.

Jias LM, Ellison G (1990) Chronic nicotine induces a specific appetite for sucrose in rats. Pharmacol Biochem Behav 35: 489-491.

Killen JD, Fortmann SP, Newman B (1990) Weight change among participants in a large sample minimal contact smoking relapse prevention trial. Addict Behav 15: 323-332.

Klesges RC, Klesges LM (1988) Cigarette smoking as a dieting strategy. Int J Eat Disord 7: 413-419.

Klesges RC Meyers AW, Klesges LM, LaVasque ME (1989) Smoking, body weight, and effects on smoking behavior: a comprehensive review of the literature. Psychol Bull 106: 204-230.

Levine LR, Rosenblatt S, Bosomworth J (1987) Use of a serotonin re-uptake inhibitor, fluoxetine, in the treatment of obesity. Int J Obes 11: 185-190.

McNair E, Bryson R (1983) Effects of nicotine on weight change and food consumption in rats. Pharmacol Biochem Behav 18: 341-344.

Pennington JAT (1989) Food Values of Portions Commonly Used (15th ed). Harper \& Row, New York.

Perkins KA, Denier CA, Mayer JA, Scott RR, Dubbert PA (1987) Weight gain associated with decreases in smoking rate and nicotine intake. Int $J$ Addict 22: 575-581.

Perkins KA, Epstein LH, Sexton JE, Pastor S (1990a) Effects of smoking cessation on consumption of alcohol and sweet, high-fat foods. J Subst Abuse 2: 287-297.

Perkins KA, Epstein LH, Stiller RL, Fernstrom MH, Sexton JE, Jacob RG (1990b) Perception and hedonics of sweet and fat taste in smokers and nonsmokers following nicotine intake. Pharmacol Biochem Behav 35: $671-676$. 
Pomerleau OF, Adkins D, Pertschuk M (1978) Predictors of outcome and recidivism in smoking-cessation treatment. Addict Behav 3: 65-70.

Rodin J (1987) Weight change following smoking cessation: the role of food intake and exercise. Addict Behav 12: $303-317$.

Shor RE, Williams DC, Cannon LK, Latta RM, Shor M (1981) Beliefs of smokers and never smokers about the motives that underlie tobacco smoking. Addict Behav 6: 317-324.

Sorensen G, Pechacek TF (1987) Attitudes toward smoking cessation among men and women. J Behav Med 10: $129-137$.

Spring B, Chiodo J, Bowen D (1987) Carbohydrates, tryptophan and behavior: a methodological review. Psychol Bull 102: 234-256.

Spring B, Wurtman J, Gleason R, Wurtman R (1991) Weight gain and withdrawal symptoms after smoking cessation: a preventive intervention using d-fenfluramine. Health Psychology 10: 216-223.

Stamford BA, Matter S, Fell RD, Papanek P (1986) Effects of smoking cessation on weight gain, metabolic rate, caloric consumption, and blood lipids. Am J Clin Nutr 43: 486-494.

Stitzer ML, Gross J (1988) Smoking relapse: The role of pharmacological and behavioral factors. In: Pomerleau OF, Pomerleau CS (Eds) Nicotine Replacement: A Critical Evaluation. Alan R. Liss, New York, pp 163-184.

Wellman PJ, Marmon MM, Reich S, Ruddle J (1986) Effects of nicotine on body weight, food intake and brown adipose tissue thermogenesis. Pharmacol Biochem Behav 24: 1605-1609. 